\title{
Quantum Frequency Translation of Single-Photon States in Photonic Crystal Fiber
}

\author{
H.J. McGuinness, ${ }^{1, *}$ M.G. Raymer ${ }^{1}$ C.J. McKinstrie, ${ }^{2}$ and S. Radic ${ }^{3}$ \\ ${ }^{1}$ Oregon Center for Optics and Department of Physics, University of Oregon, Eugene, OR USA, 97403 \\ ${ }^{2}$ Bell Labs, Alcatel-Lucent, Holmdel, New Jersey 07733 \\ ${ }^{3}$ Department of Electrical and Computer Engineering, \\ University of California at San Diego, La Jolla, CA 92093
}

(Dated: August 20, 2018)

\begin{abstract}
We experimentally demonstrate frequency translation of a nonclassical optical field via the Bragg scattering four-wave mixing process in a photonic crystal fiber (PCF). The high nonlinearity and the ability to control dispersion in PCF enable efficient translation between photon channels within the visible to-near-infrared spectral range, useful in quantum networks. Heralded single photons at $683 \mathrm{~nm}$ were translated to $659 \mathrm{~nm}$ with an efficiency of $28.6 \pm 2.2$ percent. Second-order correlation measurements on the 683-nm and 659-nm fields yielded $g_{683}^{(2)}(0)=0.21 \pm 0.02$ and $g_{659}^{(2)}(0)=0.19 \pm 0.05$ respectively, showing the nonclassical nature of both fields.
\end{abstract}

PACS numbers: 42.50.Ex, 42.65.Ky

As more advanced quantum-information applications and systems are created, it is likely that the sharing of quantum information between remote devices will be desirable, and a quantum network will be needed [1]. A good candidate to transfer such information is the single photon, which is relatively robust against loss or decoherence, allowing transfer of entanglement between remote locations. Such photons can travel long distances through optical fibers, which function optimally in particular wavelength ("telecom") ranges. Quantum frequency translation (QFT), in which a photon at one central frequency is annihilated and another photon at a different central frequency is created (see Figure 1b)), serves three important purposes in quantum networks-It allows quantum devices (memories and processors) that operate at different optical frequencies to communicate via a quantum channel; It allows low-loss, long-distance exchange over fiber between two quantum devices that operate at frequencies other than the telecom ones; It allows converting photons to frequencies where the optimal detectors operate. To be useful in quantum networks, QFT must 1) allow flexible choices of photon frequencies within the visible and near IR, 2) preserve the nature of the original state other than its central frequency, including any entanglement with other systems, and 3) must not introduce additional unwanted "noise" photons. QFT in optical fiber is predicted to satisfy all of these requirements [2]. We present the first demonstration of QFT in optical fiber, and show that it preserves the nonclassical nature of the single-photon field being translated.

Frequency conversion in second-order $\chi^{(2)}$ nonlinear optical media such as crystals via three-wave mixing has been studied in much depth, especially for coherent-state fields [3]. Sum frequency generation allows a weak field, when combined with a strong pump field, to be translated to a higher frequency (upconversion). Difference frequency generation allows for the creation of the con- a)

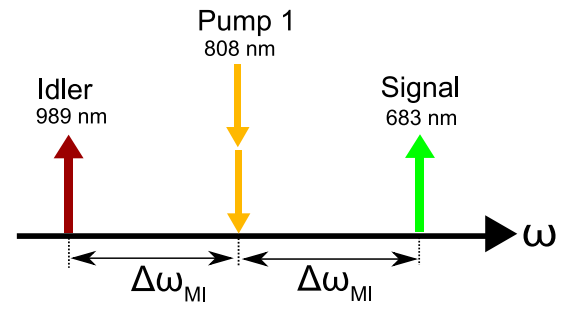

b)

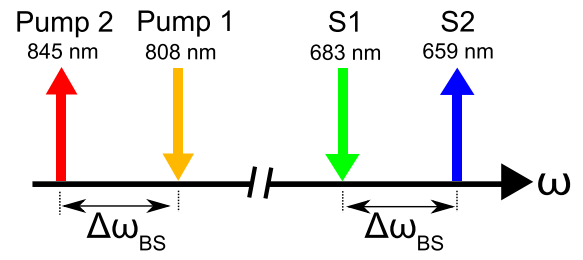

FIG. 1. (color online) a) The modulation interaction process. Two Pump 1 photons are annihilated while two sideband photons (signal and idler), equally spaced in frequency from the pump by $\Delta \omega_{\mathrm{MI}}$, are created. Up(down) arrows indicate creation(destruction). b) The Bragg-scattering quantum frequency translation process. Photons from Pump 1 and the S1 mode are annihilated while photons in Pump 2 and the $s 2$ mode are created. The frequency separation between the pumps $\Delta \omega_{\mathrm{BS}}$ is equal to the separation $\mathrm{S} 1$ and $s 2$.

jugate of a weak field, in the presence of a pump, which can either be at a higher or lower (downconversion) frequency than the original field. At the single-photon level, it has been shown that nonclassical intensity correlation between two 1064-nm fields was maintained when one beam was upconverted to $532 \mathrm{~nm}$ [4]. Also, time-energy entanglement between two fields at $1555 \mathrm{~nm}$ and $1312 \mathrm{~nm}$ was shown to be preserved after the 1312-nm field was upconverted to $712 \mathrm{~nm}$ [5]. Most recently, a single-photon state at $1300 \mathrm{~nm}$ was shown to maintain its nonclassical nature after upconversion to $710 \mathrm{~nm}$ in a periodicallypoled $\mathrm{LiNbO}_{3}$ quasi-phase-matched waveguide [6].

A disadvantage of $\chi^{(2)}$-based QFT is that the difference between the two frequencies involved must equal 
the frequency of a strong pump field, making it impractical to translate between two closely spaced channels, for example within the red portion of the spectrum. In contrast, $\chi^{(3)}$-based QFT, which we use here, requires only that two pump fields have a frequency difference equal to that by which one aims to translate the quantum state of interest. This, combined with flexible phase matching provided by photonic crystal fiber (PCF) as the medium, enables QFT within the visible-to-near-IR range, with no lower bound to the frequency separation of channels.

The process we developed for noiseless QFT is twopump Bragg-scattering (BS), a nondegenerate, four-wave mixing (FWM) process analogous to sum- or differencefrequency generation in $\chi^{(3)}$ media. As opposed to other fiber-based frequency conversion processes which involve amplification and its attendant spontaneous-emission noise, BS does not amplify the translated field, and is therefore theoretically noiseless [7]. In this sense it is also analogous to a passive beam splitter, meaning that analogues of two-photon interference apply. [8] Furthermore, it has been proved that BS is capable of translating arbitrary multi-photon states of one spectral mode to another spectral mode [7]. For classical fields, BS was first shown at telecom wavelengths [9]. Recently, several experiments have explored the BS process in achieving near-unity conversion efficiency, low-noise wavelength translation from $1545 \mathrm{~nm}$ to $1365 \mathrm{~nm}$ [10-12].

The $\chi^{(3)}$ BS process in optical fiber has additional advantages compared with sum/difference-frequency conversion in bulk and quasi-phase matched $\chi^{(2)}$ materials. Since the process occurs in optical fiber, it is straightforward to couple the translated field into another fiber with small loss. This makes translation in fiber well suited for use in quantum networks, since such networks would be sensitive to small losses. Often, the process is carried out in a single-mode fiber, where both input and output are in well defined spatial modes, which is not always the case for sum/difference generation materials. This allows the output to be more easily integrable with devices or experiments. Also, since the efficiency of the process is proportional to the product of the input power of each pump, one pump can be weak if the other pump compensate by being strong. We emphasize that PCF offers unprecedented control over the medium's dispersive properties, allowing translation to occur anywhere within the visible-to-infrared by engineering the fiber in a particular, controllable manner [13]. With the advent of hollow-core PCF [14], media other than silica maybe used for the BS interactions, adding the possibility of using more highly nonlinear materials than glass and gaining further control over dispersion of the fiber.

In this Letter we report for the first time, to our knowledge, frequency translation in optical fiber carried out with verifiably nonclassical fields. Also, to the best of our knowledge, this is the first time BS translation in a $\chi^{(3)}$ medium has been reported in the visible regime.
Briefly, a single-photon wave packet with a central wavelength $683 \mathrm{~nm}$ is created in a custom made PCF, which is then coupled into another PCF fiber along with two pump fields. The BS process takes place and with some probability (efficiency) the single-photon wave packet is translated to another wave packet with a central wavelength $659 \mathrm{~nm}$ (otherwise it is left alone). The translated and untranslated channels are then monitored with single-photon detectors which measure the degree of nonclassicality and the overall efficiency of the BS process.

The single-photon states that were translated were produced in a fiber via the one-pump FWM process called "modulation instability" (MI), shown in Figure 1a). $[15,16]$ In MI, two pump ( $p)$ photons are annihilated in the medium and two non-degenerate photons are spontaneously created. The central wavelengths of the produced "sideband" photons, also called signal (short wavelength, $s$ ) and idler (long wavelength, $i$ ), are determined by energy conservation $\left(2 \omega_{p}=\omega_{s}+\omega_{i}\right)$ and phase-matching requirements $\left(2 \beta_{p}=\beta_{s}+\beta_{i}\right)$ [17]. With a single CW laser input at power $P$, fiber nonlinearity $\gamma$ and fiber length $L$, where $P \gamma L \ll 1$, the state produced is given by

$$
|\psi\rangle \approx\left|0_{s}, 0_{i}\right\rangle+\epsilon\left|1_{s}, 1_{i}\right\rangle+\epsilon^{2}\left|2_{s}, 2_{i}\right\rangle+\ldots
$$

where $\epsilon \ll 1$ is a function of $P, \gamma$ and $L$. Since $\epsilon$ is small the state is mostly vacuum, but if the idler (signal) channel is incident upon a detector and the detector registers an event, it is very likely that the signal (idler) is in the $|1\rangle$ Fock state. This process is called heralding the signal (idler). For pulsed pump input the state is more complicated, but is not crucial for understanding the relevant physics in this Letter $[18,19]$. Phase matching in the PCF that we used in this experiment allows continuous tuning of the sidebands over a wide range (Figure 2).

After single-photon wave packets are created, one is sent into a second fiber for QFT via BS [15]. Two pump fields $p 1$ and $p 2$ and the signal field $s 1$ are coupled into the fiber, leading to the annihilation of the signal field and creation of a translated signal field $s 2$, as shown in Figure 1b). The fields must obey energy conservation $\left(\omega_{p 1}+\omega_{s 1}=\omega_{s 2}+\omega_{p 2}\right)$ and be phase-matched $\left(\beta_{p 1}+\beta_{s 1}=\right.$ $\left.\beta_{s 2}+\beta_{p 2}\right)$ for efficient translation to occur. Treating the pumps as classical fields and the signals as quantum fields, the process is governed by the Hamiltonian [2]

$$
H=\delta\left(a_{s 1}^{\dagger} a_{s 1}+a_{s 2}^{\dagger} a_{s 2}\right)+\kappa a_{s 1}^{\dagger} a_{s 2}+\kappa^{*} a_{s 2}^{\dagger} a_{s 1}
$$

where $a^{\dagger}$ and $a$ are creation and annihilation operators. The quantities $\delta$ and $\kappa$ relate to the dispersion mismatch between pumps and sidebands, and the effective nonlinearity, respectively. Utilizing the spatial equations-ofmotion $d_{z} a_{j}=i\left[a_{j}, H\right]$, the operators can be solved as functions of position along the fiber, yielding

$$
\begin{aligned}
& a_{s 1}(z)=\mu(z) a_{s 1}(0)+\nu(z) a_{s 2}(0) \\
& a_{s 2}(z)=-\nu^{*}(z) a_{s 1}(0)+\mu^{*}(z) a_{s 2}(0),
\end{aligned}
$$




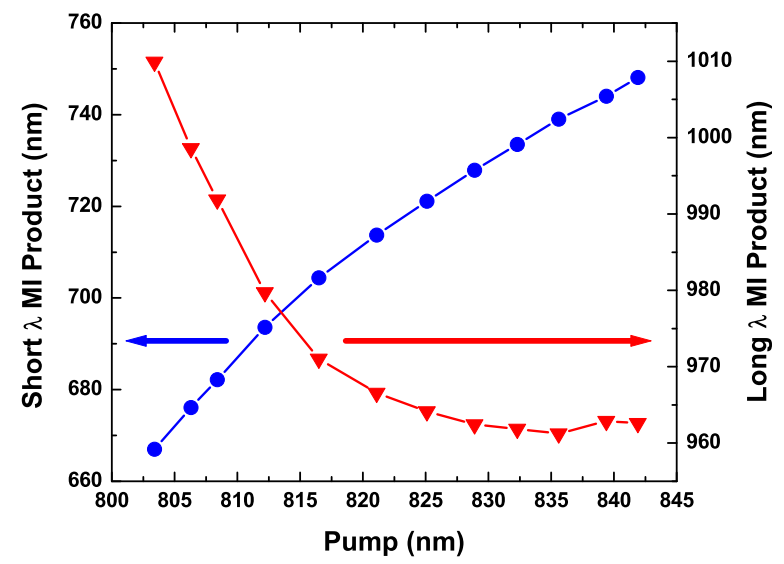

FIG. 2. (color online) Experimental data concerning the MI process in Fiber 1. The short (blue circle) and long (red triangle) wavelength sidebands refer to the signal and idler fields, respectively.

where the transfer functions $\mu$ and $\nu$ are

$$
\begin{aligned}
\mu(z) & =\cos (k z)+i \delta \sin (k z) / k \\
\nu(z) & =i \kappa \sin (k z) / k
\end{aligned}
$$

and where $k=\left(|\kappa|^{2}+\delta^{2}\right)^{1 / 2}$ and $|\mu|^{2}+|\nu|^{2}=1$. The process conserves total photon number, like a beam splitter. If the parameters are set such that $|\nu(L)|=1$, then all of the signal field will be translated to the idler field by the end of the fiber.

The above analysis is valid for single-mode, CW fields. For pulsed fields a more general theory is appropriate [8]. The temporal and spectral properties of all input fields play a significant role in determining the nature of the translated field. For efficient translation, temporal overlap of the fields must be maximized and care must be taken that the bandwidth of the input field is not greater than the bandwidth of the BS process, which is a function of fiber dispersion, length and pump bandwidth.

In order to demonstrate that the $s 1$ input and the $s 2$ output are nonclassical, the conditional second-order degree of coherence $g^{(2)}(0)$ is measured for these fields. A result of $g^{(2)}(0)<1$ indicates a nonclassical field [20]. For a heralded field, $g^{(2)}(0)$ is given experimentally by

$$
g^{(2)}(0)=\frac{N_{A B C} N_{C}}{N_{A C} N_{B C}}
$$

where $A$ and $B$ label the detectors monitoring the output of the heralded signal field, and $C$ labels the heralding detector in the idler field. The quantities $N_{i}, N_{i j}, N_{i j k}$ are the number of single, double coincidence, triple coincidence events between detectors $i, j$ and $k$ over the time interval of the data collection, respectively [21].

The experimental apparatus is shown in Figure 3. Pump 1 and Pump 2 were 100 ps titanium-sapphire lasers operating at $808 \mathrm{~nm}$ and $845 \mathrm{~nm}$, respectively. Both lasers, having repetition rates of $76 \mathrm{MHz}$, were long-pass filtered to extinguish any light in the quantum channels. A small percentage of Pump 1 was split off and sent to 32 $\mathrm{m}$ of a custom-made solid-core PCF, denoted as Fiber 1, having a zero-dispersion wavelength (ZDW) at approximately $796 \mathrm{~nm}$. Sidebands were created at $683 \mathrm{~nm}$ and $989 \mathrm{~nm}$ using the MI process in which phase matching was achieved by using the fiber birefringence [22, 23]. These beams were split, with the $989-n m$ idler being sent to a single-photon detector for heralding. Both sidebands were filtered with 13-nm bandpass filters to remove unwanted spontaneous Raman scattering.

The rest of Pump 1, and all of Pump 2, were combined on a dichroic mirror, with the resulting beam further combined with the $683-\mathrm{nm}$ signal from Fiber 1 by use of another dichroic mirror. The pulses of both pumps and the $683-\mathrm{nm}$ signal pulse were overlapped temporally. All beams had the same linear polarization. All three beams were incident upon 20 meters of Fiber 2 (Crystal Fibre, model number NL-PM-750), and aligned on one of the principal birefringence axes of this fiber. Around 20(30) mW from Pump 1(2) was coupled into Fiber 2. We achieved over 31 percent coupling of the $683-\mathrm{nm}$ beam from Fiber 1 into Fiber 2 prior to translation. The state of the 683-nm field was frequency translated with a certain probability to the field centered around $659 \mathrm{~nm}$. After exiting Fiber 2, the pump components were filtered out of the beam, which was then separated into the 683$\mathrm{nm}$ and $659-\mathrm{nm}$ channels. Both channels were filtered with 13-nm bandpass filters. Conditional second-order correlation measurements $\left(g^{(2)}(0)\right)$ of the $683-\mathrm{nm}$ and 659-nm channels were carried out in coincidence with the 989-nm MI idler channel.

To verify that the translation process is occurring with high efficiency, we measured both the depletion efficiency of the $683-\mathrm{nm} s 1$ input and the creation efficiency of the $659-\mathrm{nm} s 2$ output, which should be equal. The depletion efficiency was obtained by measuring the rates of counts (minus pump noise counts) in the output $683-\mathrm{nm}$ $s 1$ channel with and without the pump beams coupled into Fiber 2. The depletion efficiency equals one minus the ratio of these two count rates. The result, $28.6 \pm 2.2$ percent, is not dependent on comparing quantum efficiencies of two detectors. To measure the creation efficiency of the $659-\mathrm{nm} s 2$ channel, the count rate in this channel was monitored with and without the pumps. When detector efficiencies are taken into account, this measurement yields $29.4 \pm 2.4$ percent, agreeing with the depletion measurement to within error.

To show the nonclassical nature of the translated and untranslated light, $g^{(2)}(0)$ measurements were performed on both the $683-\mathrm{nm}$ and $659-\mathrm{nm}$ channels at the output of the fiber, yielding $0.21 \pm 0.02$ and $0.19 \pm 0.05$, respectively. To determine the average value and error of the $g^{(2)}(0)$ measurements, 30 runs of the experiment lasting 20 seconds each were performed. 


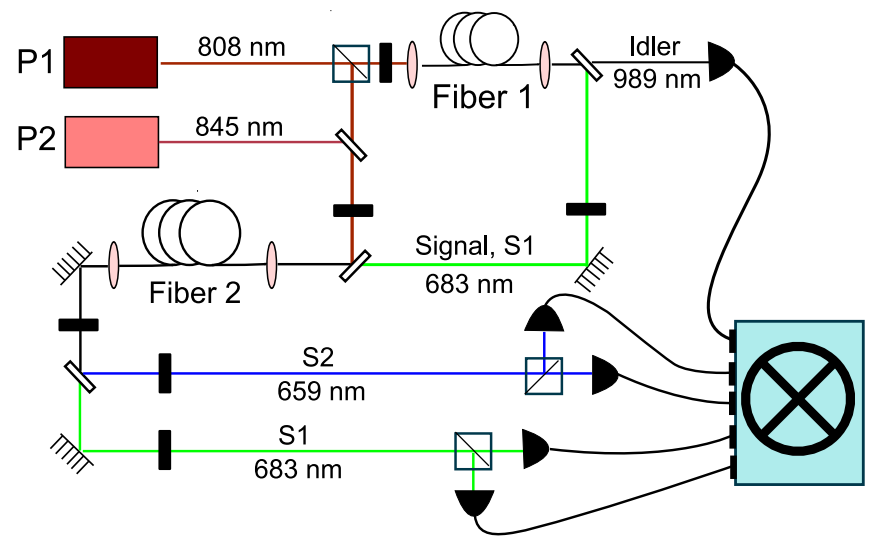

FIG. 3. (color online) Setup used to create and frequency translate single-photon states. Both pumps $p 1$ and $p 2$ are 100 ps titanium sapphire lasers. A single photon is created in Fiber one at $683-\mathrm{nm}$, heralded, and combined with $p 1$ and $p 2$ to be translated to $659-\mathrm{nm}$ in Fiber two. Five detectors measure $g^{(2)}(0)$ of both 683-nm and 659-nm channels, with the $989-\mathrm{nm}$ channel detector acting as the herald. Opaque rectangles represent either longpass or bandpass filters and transparent rectangles signify dichroic mirrors.

There are several factors which cause the measured values of $g^{(2)}(0)$ to be nonzero. After heralding, the photon state to be translated will be predominantly the $|1\rangle$ Fock state. But the state will also include small amplitudes for number states above 1 , which will increase the value of $g^{(2)}(0)$. For states generated via heralded MI there will always be a trade-off between higher count rates and high single-photon state probability (unless a numberresolving detector is used for heralding). Low detector efficiency (12 percent) for the 989-nm channel demanded higher input power than desired. Pump 1 input power in Fiber 1 was chosen to give reasonably high photon flux and coincident count rates while guaranteeing the heralded state (1) was predominantly the $|1\rangle$ state. Another possible cause for increased $g^{(2)}(0)$ was from accidental coincidence counts from Raman scattering, detector dark counts and other noise. Over a given time, an experiment that counted $N_{i}$ idler counts and $N_{s}$ signal counts, both which derived from $N_{p}$ pulses, would expect to register $N_{i} N_{s} / N_{p}$ accidental coincidences if the idler and signal beams were independent and poisson. Therefore any measurement that depends on coincidence counts from a correlated source, such as sideband creation via MI, needs to register coincidences above this value. In our experiment the coincidence level was approximately 8.2 times higher than the expected accidental coincidence value for coincidences between the $683-\mathrm{nm}$ channel and the $989-\mathrm{nm}$ heralding channel. The coincidence level for the $659-\mathrm{nm}$ translated channel was approximately 6.5 times higher than the expected accidental coincidences.

These same noise counts affected the measured efficiency of the translation process. Approximately 24 per- cent of counts in the 659-nm channel, and 11 percent in the $683-\mathrm{nm}$ channel, were from pump noise (e.g., Raman scattering). Most of the noise originated from the 808$\mathrm{nm}$ pump. It is clear how such noise could be drastically reduced. Due to the wavelength range of the available pumps, and the dispersion characteristics of the fibers, it was necessary for Pump 1 to operate at $808 \mathrm{~nm}$, which is only 50 to $70 \mathrm{~nm}$ away from Fiber 2's ZDW. Many nonlinear effects grow exponentially stronger the closer the pump field wavelength is to the fiber's ZDW. Operating the pump farther from the ZDW, and farther from the signal and idler modes in general, would strongly decrease the noise in these channels. Also, the Stokes and antiStokes Raman noise can be strongly reduced by cooling Fiber 2 to liquid nitrogen temperature [24].

Theory predicts that BS is capable of 100 percent translation efficiency of arbitrary states [7]. In our experiment various factors limit the efficiency. The most likely factor was the relatively large spectral width of the 683$\mathrm{nm}$ field created by MI. This conjecture is supported by the fact that the full width at half maximum (FWHM)of the output translated field was less than that of the input untranslated state, indicating the translation process had a narrower acceptance bandwidth than the bandwidth of the input field. These widths were measured using an input signal at $683-\mathrm{nm}$ created by high-gain MI, with a FWHM of approximately $2.0 \mathrm{~nm}$, while the translated field had a FWHM equal or less than $1.5 \mathrm{~nm}$, the minimum width resolution of the spectrometer. The wide FWHM of the input field was due to Pump 1 being close in wavelength to the ZDW of Fiber $1[19,25]$. For this experiment it was unfortunately necessary for the pump and ZDW of Fiber 1 to be close in wavelength, but this is not indicative of a fundamental limitation of the BS translation process.

We have demonstrated frequency translation of nonclassical states of light via the four-wave mixing Bragg scattering process. This is the first time BS on the quantum level in a $\chi^{(3)}$ media has been reported. This is also the first time translation within the visible regime has been reported. While translation in $\chi^{(3)}$ media requires two pumps, as opposed to translation in $\chi^{(2)}$ media, which requires only one, there are a number of advantages such a method offers. Because the translating device could be easily coupled to an optical fiber network, it is the clear choice for translation in a quantum network. The two-pump configuration allows for a more flexible achievement of phase-matching conditions, allowing translating between spectrally close channels. This in turn opens possibilities for two-photon (Hong-OuMandel) interference between photons of different colors [8] and ultimately linear-optics quantum computing [26] using multiple frequency channels.

We thank S. van Enk for helpful comments. This work was supported by NSF Grant ECCS-0802109. 
* hmcguinn@uoregon.edu

[1] J. I. Cirac et al., Phys. Rev. Lett., 78, 3221 (1997).

[2] C. J. McKinstrie et al., Opt. Express, 13, 9131 (2005).

[3] R. W. Boyd, Nonlinear Optics (Academic, New York, 2008) Chap. 2.

[4] J. M. Huang and P. Kumar, Phys. Rev. Lett., 68, 2153 (1992).

[5] S. Tanzilli et al., Nature, 437, 116 (2005).

[6] M. T. Rakher et al., arXiv:1004.2686v1 [quant-ph].

[7] C. J. McKinstrie et al., Opt. Express, 12, 5037 (2004).

[8] M. G. Raymer et al., Opt. Commun., 283, 747 (2010).

[9] K. Inoue, IEEE Photon. Technol. Lett., 6, 1451 (1994).

[10] K. Uesaka et al., IEEE J. Sel. Top. Quantum Electron., 8, 560 (2002).

[11] A. H. Gnauck et al., Opt. Express, 14, 8989 (2006).
[12] D. Mechin et al., Opt. Express, 14, 8995 (2006).

[13] A. Birks et al., Opt. Lett., 22, 961 (1997).

[14] R. F. Cregan et al., Science, 285, 1537 (1999).

[15] C. J. McKinstrie et al., IEEE J. Sel. Top. Qu. Elect., 8, 538 (2002).

[16] J. Fulconis et al., Opt. Express, 13, 7572 (2005).

[17] G. P. Agrawal, Nonlinear Fiber Optics: 4th edition (Academic, San Diego, 2006).

[18] J. Chen et al., Phys. Rev. A, 72, 033801 (2005).

[19] K. Garay-Palmett et al., Opt. Express, 15, 14870 (2007).

[20] R. Loudon, The Quantum Theory of Light: 3rd edition (Oxford University, Oxford, 2000) Chap. 5.

[21] M. Beck, J. Opt. Soc. Am. B, 24, 2972 (2007).

[22] J. Fan and A. Migdall, Opt. Express, 13, 5777 (2005).

[23] B. J. Smith et al., Opt. Express, 17, 23589 (2009).

[24] H. Takesue and K. Inoue, Opt. Express, 13, 7832 (2005).

[25] M. E. Marhic et al., IEEE J. Sel. Top. Quantum Electron., 10, 1133 (2004-2012).

[26] E. Knill et al., Nature, 409, 46 (2001). 